\title{
Quantitative evaluation of healthy epidermis by means of multiphoton microscopy and fluorescence lifetime imaging microscopy
}

\author{
Elisa Benati ${ }^{1}$, Valerio Bellini ${ }^{2}$, Stefania Borsari ${ }^{1}$, Christopher Dunsby $^{3}$, Chiara Ferrari ${ }^{1}$, \\ Paul French ${ }^{3}$, Mario Guanti ${ }^{1}$, Davide Guardoli ${ }^{1}$, Karsten Koenig ${ }^{4}$, Giovanni Pellacani ${ }^{1}$, \\ Giovanni Ponti ${ }^{1}$, Simona Schianchi ${ }^{1}$, Clifford Talbot ${ }^{3}$ and Stefania Seidenari ${ }^{1}$ \\ ${ }^{1}$ Department of Dermatology, University of Modena and Reggio Emilia, Modena, Italy, ${ }^{2}$ CNR Institute of NanoSciences S3, Modena, Italy, ${ }^{3}$ Photonics \\ Group, Department of Physics, Imperial College London, London, UK, and ${ }^{4}$ Faculty of Physics \& Mechatronics, Saarland University, \\ Saarbrücken, Germany
}

Background/purpose: Multiphoton microscopy (MPM) enables the assessment of unstained living biological tissue with submicron resolution, whereas fluorescence lifetime imaging microscopy (FLIM) generates image contrast between different states of tissue characterized by various fluorescence decay rates. The aim of this study was to compare the healthy skin of young individuals with that of older subjects, as well as to assess the skin at different body sites, by means of MPM and FLIM.

Methods: Nineteen elderly patients were examined on the outer side of the forearm, whereas 30 young individuals were assessed on the dorsal and volar sides of the forearm and on the thigh.

Results: Cell and nucleus diameters, cell density and FLIM vary according to the epidermal cell depth and the skin site. In elderly subjects, epidermal cells show morphologic al- terations in shape and size, with smaller cell and nucleus diameters; the number of basal cells is decreased, whereas the mean fluorescence lifetimes at both the upper and the lower layers increase.

Conclusion: This study provides quantitative and qualitative data on normal epidermis at different skin sites at different ages and represents a reference for the clinician attempting to understand the effectiveness of MPM and FLIM in discriminating diseased states of the skin from normal ones.

Key words: skin morphology - fluorescence lifetime imaging - skin ageing - high-resolution imaging

(C) 2011 John Wiley \& Sons A/S

Accepted for publication 20 November 2010
$\mathrm{T}^{\mathrm{H}}$ HE DEVELOPMENT of non-invasive imaging techniques has improved the clinical diagnosis of skin diseases. Recently, multiphoton microscopy (MPM) has emerged as a minimally invasive biomedical imaging modality that holds great potential for dermatological applications (1). In conventional fluorescence microscopy or confocal fluorescence microscopy, fluorophores are excited by absorption of one photon of relatively high energy in the visible or the ultraviolet spectrum. In MPM, excitation of the fluorophores relies on the simultaneous absorption of two or more photons of lower energy in longer wavelengths, usually in the near-infrared spectrum. As the endogenous fluorophores including keratin, $\mathrm{NAD}(\mathrm{P}) \mathrm{H}$, elastic fibres, etc., can be efficiently excited by MPM $(2,3)$, the combination of autofluorescence and second harmonic generation in
MPM imaging can provide morphological, structural and chemical information concerning both the cells and the extracellular matrix of skin without the need for extrinsic labeling $(4,5)$. Masters and colleagues, in 1997, published one of the first studies on multiphoton laser scanning microscopy on human skin. They visualized the cellular structures of the epidermis and the upper dermis by placing the forearm of one of the authors beneath their microscope $(3,6,7)$. In addition to morphology, the authors also presented the spectral measurements of skin autofluorescence, supporting the concept that $\mathrm{NAD}(\mathrm{P}) \mathrm{H}$ is the primary source of autofluorescence in the epidermis. Over the past decade, multiphoton imaging has found a variety of applications in dermatology, including the study of tumours and inflammatory disorders and 
in situ drug screening (1, 3, 7-10). The timeresolved analysis of the fluorescence signal, known as fluorescence lifetime imaging microscopy (FLIM), is a non-invasive imaging technique that generates image contrast between different states of tissue characterized by different fluorescence decay rates. In addition to information on cell and tissue morphology provided by multiphoton intensity imaging, FLIM can enable different cell or fibre types to be distinguished based on their fluorescence decay profiles. It is well known that skin morphology and function vary according to different skin sites and that they are altered during the ageing process. The aim of this study was to compare the healthy skin of young individuals (20-35 years old) with that of older subjects (over 60 years), as well as to assess the skin at different body sites by means of multiphoton FLIM. The cell morphology, fluorescence intensity and lifetime of upper and lower layers of the epidermis were analysed.

\section{Material and Methods}

Instrumentation and acquisition of data Multiphoton tomography

The imaging system DermaInspect ${ }^{\circledR}$ (Jenlab GmbH, Jena, Germany), is an in vivo multiphoton tomograph system with a total dimension of $75 \times 120 \times$ $140 \mathrm{~cm}^{3}$, is mounted on a transportable workstation (8) and provides intra-tissue scanning with subcellular spatial resolution (horizontal $<1 \mu \mathrm{m}$, vertical $<2 \mu \mathrm{m}$ ). This CE-marked and commercially available imaging tool is rated as a class $1 \mathrm{M}$ device according to the European laser safety regulations and consists of three major modules: the laser, a scanning-detector unit and a control module. The laser source utilizes a mode-locked $80 \mathrm{MHz} \mathrm{Ti}: \mathrm{S}$ (titanium:sapphire) laser (Mai-Tai; Spectra Physics, Mountain View, CA, USA) with a tuning range of $710-920 \mathrm{~nm}$, $75 \mathrm{fs}$ pulse width and a maximum laser power of $50 \mathrm{~mW}$ at the sample. The scanning-detector unit consists of a trigger diode, fast $x-y$ galvo scanners, beam expander and scan optics, a $40 \times$ focusing optics (NA $=1.3$ oil) as well as two photomultiplier tubes: one for intensity imaging and one for FLIM. A coloured-glass BG39 filter is used to block the scattered laser radiation light.

\section{FLIM}

FLIM was implemented in the DermaInspect ${ }^{\circledR}$ system using a time-correlated single photon counting module (Becker \& Hickl SPC830, Becker \& Hickl GmbH, Berlin, Germany), which had a temporal instrument response function (IRF) of approximately $250 \mathrm{ps}$ in width. The emitted autofluorescence was spectrally selected by means of a short-pass optical filter in order to protect the detector (a PMH 100-1, Becker \& Hickl $\mathrm{GmbH}$ ) from any scattered excitation light. False-colour lifetime maps were produced by assigning a colour to each image pixel according to its lifetime value. For the calculation of FLIM images, we used the software SPCIMAGE from Becker \& Hickl GmbH with the 'binning' factor set to 2 and a single exponential decay was fitted in order to calculate the mean decay rate for each pixel. An IRF was acquired on each day from second harmonic generation from a sample of urea crystals dried onto a microscope coverglass. The resulting IRF was then imported into SPCImage before the decay analysis. Following the calculation of the FLIM maps, a 'FLIM analysis assistant' macro written using Autolt was used to extract the total fluorescence intensity and photon-weighted fluorescence lifetime histograms for selected regions of interest (ROI), e.g. the cytoplasm of a single cell. The 'FLIM analysis assistant' also calculated the mean fluorescence intensity and lifetime for each ROI. For the processing of MPM images, a standardized area of $100 \times 100 \mu \mathrm{m}$ was selected within each image. The total number of cells and the diameters of the cell cytoplasm and the nucleus were measured in this area. For each fluorescence intensity and lifetime image, average values were calculated for over five ROI corresponding to five individual cells (11). Figure 1 illustrates the ability of FLIM to provide additional contrast compared with fluorescence intensity images. We believe that cells represented in the FLIM maps with red having low fluorescence lifetimes are melanocytes and melanin-containing keratinocytes, while cells represented in green with longer lifetimes are keratinocytes, and that these appear clearly distinguished in the FLIM map. This is supported by previous work by Dimitrow et al. (11) and previous publications showing that the fluorescence decay of melanin can be described by a triple exponential decay with a significant contribution from a fast 200 ps decay component (12), which is shorter than the mean lifetime of cytosolic NAD(P)H (measured to be 950 ps in rat hippocampal slices ex vivo) (13). 

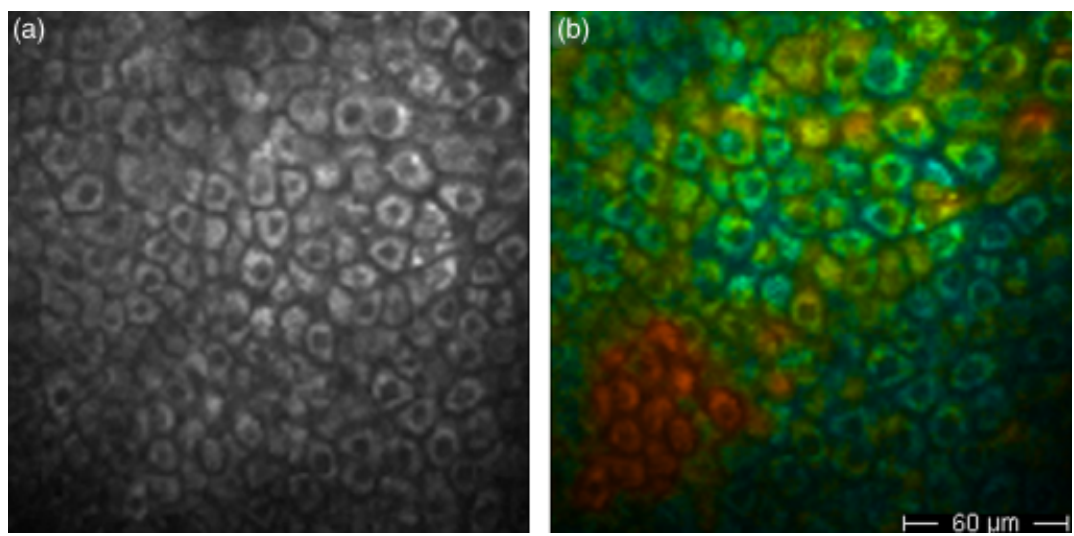

Fig. 1. (a) Intensity image of epidermal cells, (b) corresponding FLIM image, where melanocytes and melanin-containing cells are represented in red, whereas keratinocytes not containing melanin, showing higher fluorescence lifetime values, are depicted in green. The false colour scale represents the fluorescence lifetime on a scale of 0 (red)-2000 (blue) ps.

\section{Image acquisition and analysis}

For in vivo examination, a drop of water was placed between the skin and the microscope cover glass, which is fixed onto a metal coupler by a ring of adhesive tape. The metal coupler was then attached to the MPM system. The desired imaging depth was found by adjusting the focus while viewing the fluorescence intensity image in real time using the DermaInspect ${ }^{\circledR}$ control software (JLSCAN_1100). The imaging depth was first pinpointed to the upper layer (stratum corneum); afterwards, a stepwise descent from the surface to a depth of approximately $200 \mu \mathrm{m}$ was performed at intervals of $15 \mu \mathrm{m}$. Each optical section, consisting of $512 \times 512$ pixels, was taken with a scanning time of $25.5^{\circ} \mathrm{s} /$ frame. The JLSCAN_1100 software image parameters were set to 'contrast' $^{\prime} 930$ and 'brightness' $=530$, and an attenuator power between 20 and $45 \mathrm{~mW}$. For selective imaging and fluorescence lifetime measurements of the epidermis, an excitation wavelength of $760 \mathrm{~nm}$ was used. Images were analysed using the software SPCIMAGE (Becker \& Hickl $\mathrm{GmbH}$ ), in order to produce the fluorescence lifetime image for each skin layer. The maximum imaging depth corresponds to the working distance of the microscope objective used by the DermaInspect ${ }^{\circledR}$ system $(200 \mu \mathrm{m})$.

\section{Case Study}

The skin of 49 subjects aged 20-90 was investigated at three different skin sites by MPM. Nineteen elderly patients (over 60) were examined on the outer side of the forearm, whereas 30 young individuals (20-35 years old) were assessed on the dorsal and volar sides of the forearm and on the thigh. We also obtained FLIM images of 40 of these subjects. The upper epidermal and the lower epidermal layers, corresponding to the granulosum and the spinosum/basal layer, respectively, were considered separately.

\section{Statistics}

Statistical examinations were performed using SPSS 12.0 for Windows (SPSS Inc., Chicago, IL, USA). The mean values and standard deviations were calculated for each considered parameter. Data referring to different skin sites and different age groups were evaluated by means of the Mann-Whitney test. A $P$ value $\leq 0.05$ was considered statistically significant.

\section{Results}

Table 1 shows the results of cell and nucleus diameter measurements on the images obtained from different areas in 20-35-year-old individuals. The upper epidermal and lower epidermal layers, corresponding to the granulosum and the spinosum/basal layer, respectively, are considered separately. Cell and nucleus diameters are higher in the upper layers, whereas the number of cells is lower, indicating that cells in the lower layers are small and closely packed. The ratio of diameters for the nucleus/cytoplasm is higher at the lower levels, indicating that the nucleus is proportionally larger in the lower ones. With respect to the volar forearm and the thigh, on the dorsal forearm, cell and nucleus diameters are larger at the upper layers and smaller at the lower layers. The number of cells in a $100 \times 100 \mu \mathrm{m}$ area is lower on the thigh, both at 
TABLE1. Comparison between different areas in thirty 20-35-year-old subjects: cell and nucleus diameter, number of cells in a region of interest of $100 \times 100 \mu \mathrm{m}$, cytoplasm/nucleus intensity ratios and fluorescence lifetime values in 21 subjects

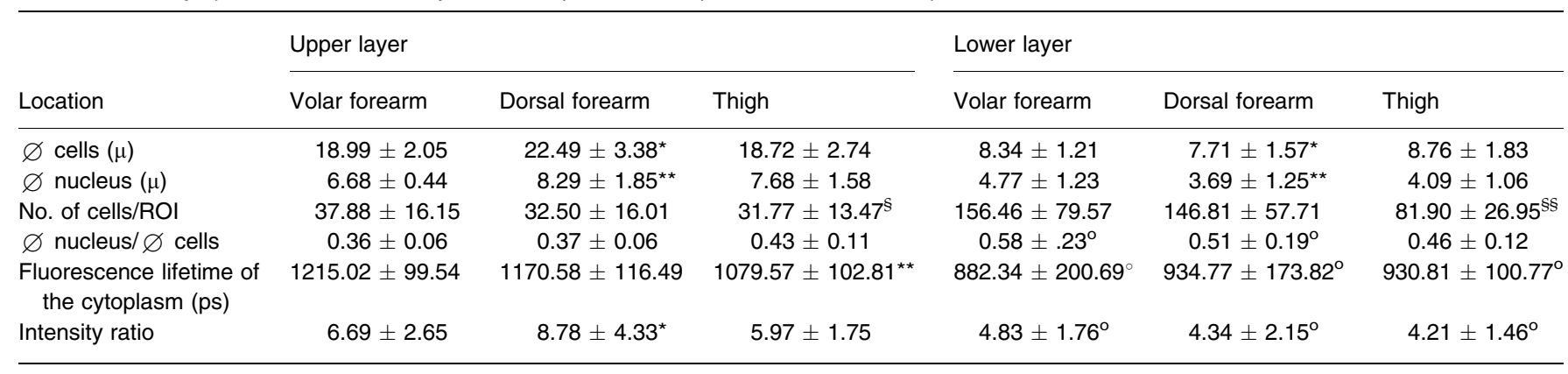

*Significant vs. volar forearm and thigh at the upper layer.

**Significant vs. volar forearm at the upper layer.

${ }^{\text {s }}$ Significant vs. volar forearm at the upper layer.

${ }^{\$}{ }_{S}$ Significant vs. volar forearm and dorsal forearm at the lower layer.

${ }^{\circ}$ Significant vs. the same site at the upper layer.

$\varnothing$, diameter; ROI, region of interest; intensity ratio, cytoplasm intensity/nucleus intensity.

the upper and at the lower layers. The fluorescence lifetime of the cytoplasm is shorter at the lower epidermal level, and on the thigh at the upper layer too, compared with the dorsal and volar forearms. The cytoplasm/nucleus intensity ratio is smaller at the lower layers, as at that level, the cytoplasm is less fluorescent. Finally, on the dorsal forearm, the intensity of the cytoplasm is higher at the upper layer and lower at the lower one compared with the other skin sites. Significances are indicated in Table 1.

Figure 2 shows the FLIM images of the upper and lower layers at the dorsal forearm, volar forearm and thigh. Cells do not show morphological variations according to the skin site. Keratinocytes are represented in green, whereas red cells or structures correspond to melanocytes and pigmented keratinocytes or melanosomes, respectively. Table 2 shows a comparison between the epidermis of young and elderly subjects. Both cell and nucleus diameters are smaller at the upper layers in the elderly. The number of basal cells is also decreased in the elderly. The FLIM values at both the upper and lower layers increase during the ageing process. Significant differences are indicated in Table 2. In elderly individuals, cells appear with undefined and irregular contours especially at the upper layers (Fig. 3). The intercellular spaces also seem larger, as, in spite of a smaller cell size in the elderly, the number of cells $/ 100 \times 100 \mu \mathrm{m}$ is equal or lower. These aspects are not yet quantifiable due to the lack of appropriate software that allows the assessment of cell morphology.

\section{Discussion}

The epidermis is the outermost portion of the skin and is composed of stratified squamous epithelium. The epidermal thickness varies from $50 \mu \mathrm{m}$ on the eyelids to $1.5 \mathrm{~mm}$ on the palms and soles. The innermost layer of the epidermis consists of a single layer of cuboidal cells called basal cells, which differentiate and migrate towards the skin surface and finally form the cornified layer of the stratum corneum, which is composed of flattened and dead cells. The epidermis protects our body from desiccation even in a dry environment, as well as from external invasion of injurious agents. The stratum corneum also plays an important role by binding water to keep the skin surface smooth and flexible even in an extremely dry environment (14). Epidermal function can be easily investigated by means of numerous noninvasive techniques, assessing its electrical properties related to water content, water loss through the epidermis (TEWL), microcirculation, elasticity, etc. On the contrary, the morphology of the epidermis in vivo is difficult to assess due to limitations in the resolution of the optical systems used or the potential health hazards of invasive methods. In multiphoton imaging, the morphology of corneocytes and epidermal keratinocytes can be obtained without fixation and staining procedures, due to the presence of keratin autofluorescence $(3,6,7,15-18)$. Healthy epidermis shows a homogenous distribution of cells that are divided by thin non-fluorescent intercellular spaces. As nuclei are deficient in autofluores- 

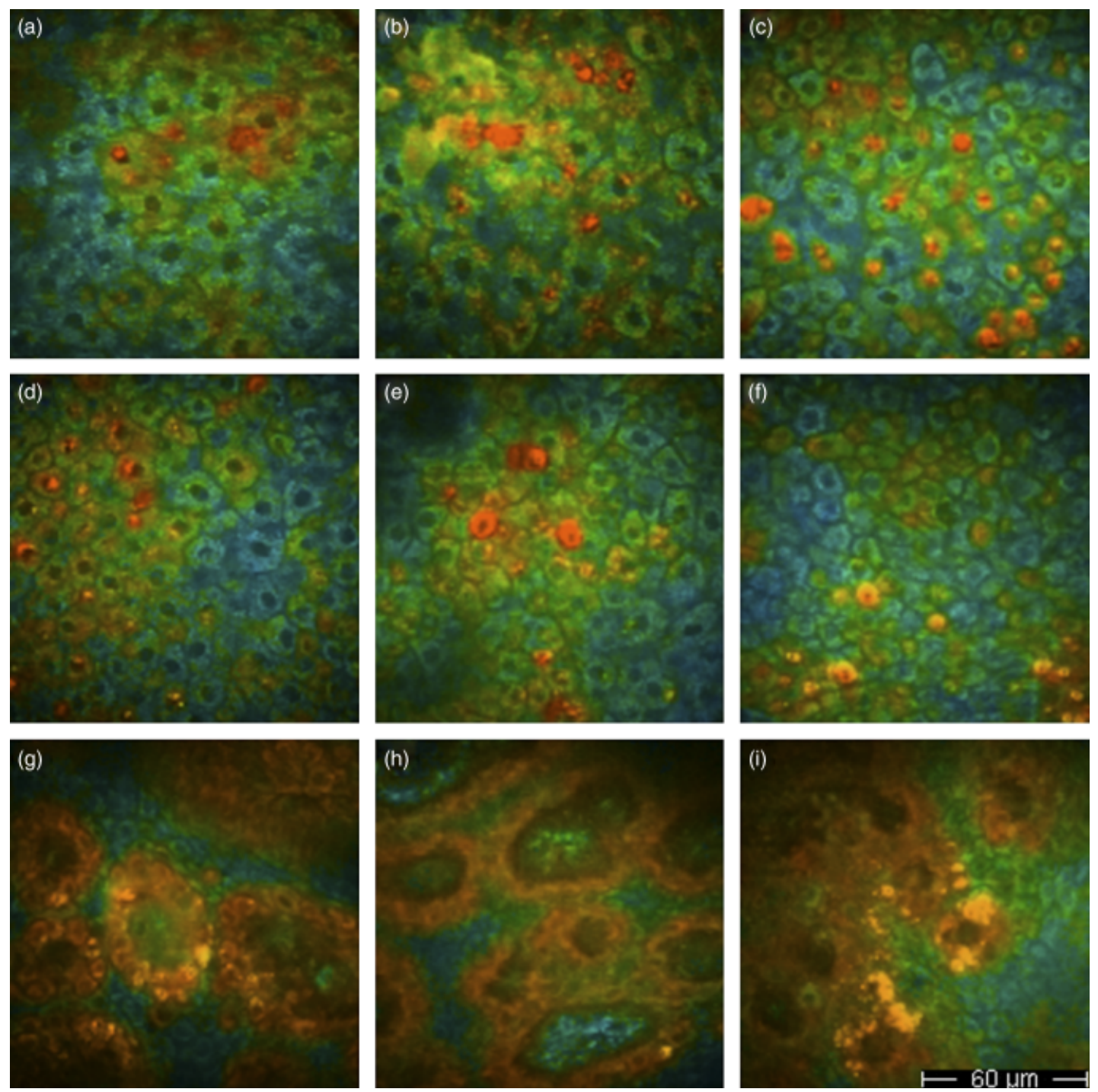

Fig. 2. $(a, b, c)$ FLIM images of the upper epidermal layers; ( $a$ ) dorsal forearm, $(b)$ volar forearm, $(c)$ thigh; $(d, e, f)$ FLIM images of lower epidermal layers; $(d)$ dorsal forearm, (e) volar forearm, $(f)$ thigh; keratinocutes show a smaller size with respect to the upper layer; $(g, h, i)$ FLIM images of the dermal-epidermal junction; $(g)$ dorsal forearm, $(h)$ volar forearm, $(i)$ thigh; the tips of the dermal papillae are surrounded by melanin-containing cells (in red), which show lower FLIM values with respect to keratinocytes (in green). The false colour scale represents the fluorescence lifetime on a scale of 0 (red)-2000 (blue) ps.

TABLE 2. Comparison between young and elderly individuals at the dorsal forearm: cell and nucleus diameters, number of cells in a region of interest of $100 \times 100 \mu \mathrm{m}$ in 30 young and 19 elderly subjects, and fluorescence lifetime values referring to 21 young and 19 elderly subjects

\begin{tabular}{|c|c|c|c|c|}
\hline & \multicolumn{2}{|l|}{ 20-35 years } & \multicolumn{2}{|l|}{$>60$ years } \\
\hline$\varnothing$ cells & $22.49 \pm 3.38$ & $7.71 \pm 1.57$ & $20.87 \pm 3.26^{*}$ & 7.521 .77 \\
\hline$\varnothing$ nucleus & $8.29 \pm 1.85$ & $3.69 \pm 1.25$ & $6.64 \pm 1.01^{*}$ & $3.61 \pm 1.00$ \\
\hline No. of cells/ROI & $32.50 \pm 16.01$ & $146.81 \pm 57.71$ & $32.20 \pm 8.64$ & $130.50 \pm 61.59^{\star}$ \\
\hline Fluorescence lifetime of the cytoplasm (ps) & $1170.58 \pm 116.49$ & $934.77 \pm 173.82$ & $1279.84 \pm 85.14^{*}$ & $1144.79 \pm 167.05^{\star}$ \\
\hline
\end{tabular}

*Significant vs. corresponding 25-35 values.

$\varnothing$, diameter; ROI, region of interest.

cence signals, they appear as dark areas inside the fluorescent cytoplasm (Fig. 4). The stratum corneum shows strong keratin-based fluorescent hexagonal-shaped flat and large cells, with no internal structuring (corneocytes). At a depth of about $20 \mu \mathrm{m}$ from skin surface, we find the 

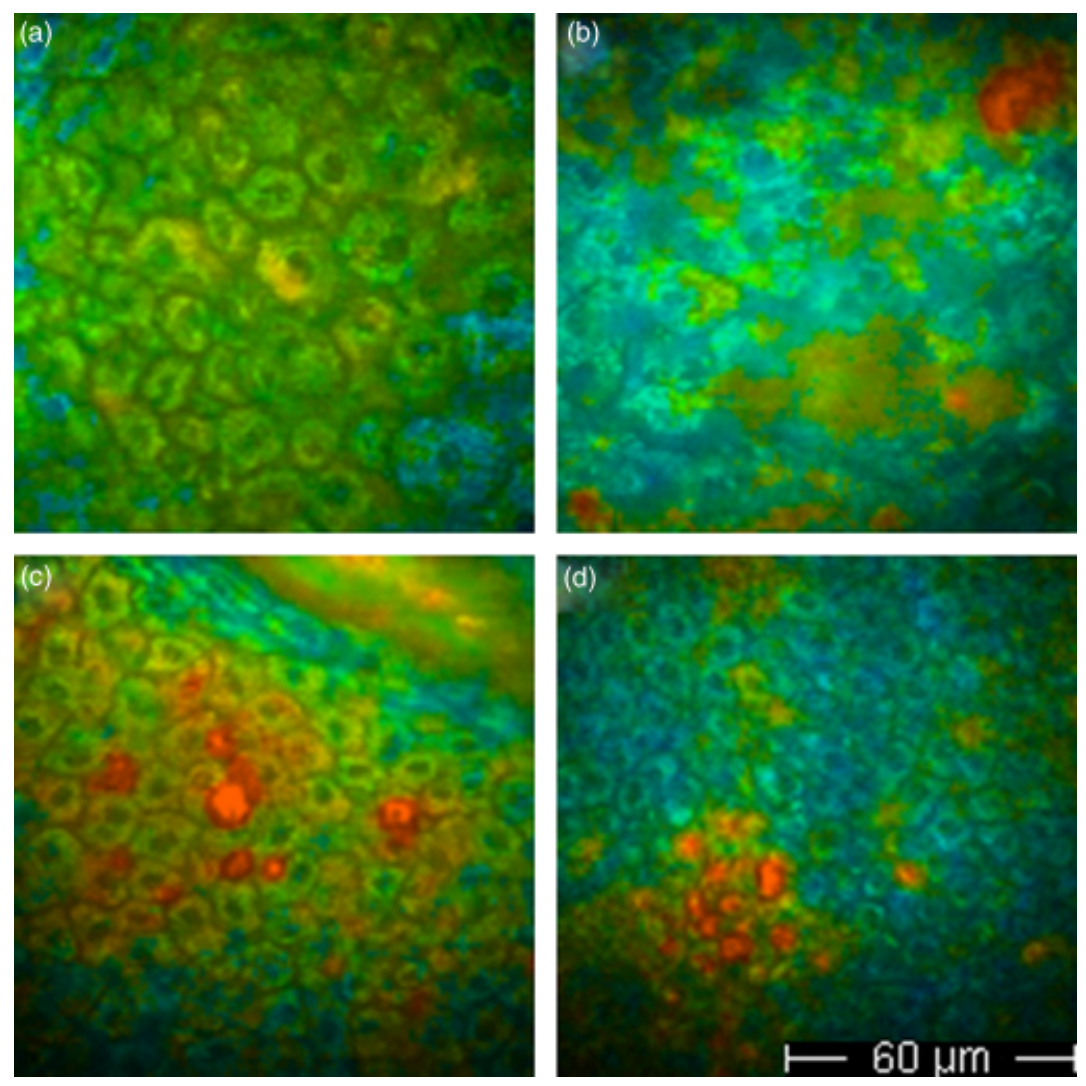

Fig. 3. (a, b) FLIM images of the dorsal forearm, upper layer;(a) in 20-35-year-old individuals, (b) in individuals over 60 years; (c, d) FLIM images of the dorsal forearm, lower layer; (c) in 20-35-year-old individuals, (d) in individuals over 60 years. The false colour scale represents the fluorescence lifetime on a scale of 0 (red)-2000 (blue) ps.

stratum granulosum, where keratinocytes appear as large, oval cells with autofluorescence in the cytoplasm and dark nuclei. Keratinocytes of the stratum granulosum are morphologically similar in shape, size and brightness. In the stratum spinosum, cell diameter decreases and cell density increases going more in depth towards the basal layer. At $50-100 \mu \mathrm{m}$ from the skin surface, we find the basal layer with brightly fluorescent small polygonal keratinocytes and dark nuclei. At the dermal-epidermal junction, dark areas with round/oval shape, interrupting the basal layer and corresponding to the top of the dermal papillae, where we can appreciate the presence of fibres, can be observed.

In this study, we provided quantitative data on cell and nucleus diameters, cell density and nucleus-cytoplasm fluorescence intensity at different levels of the epidermis at three skin areas in subjects aged 25-35 years, and showed that cell and nucleus diameters, cell density and fluorescence not only vary according to epidermal cell depth but also depending on the skin site. This study also demonstrates that fluorescence lifetime decay profiles are different in the upper and lower layers, which is most likely to be caused by the greater number of melanin-containing cells in the lower layers. This variation in the fluorescence lifetime may also indicate differences in metabolic activity at different levels of the epidermis (19), but further investigation is required in order to separate the two effects of melanin concentration and cell metabolism on the overall mean fluorescence lifetime for a single cell.

In this study, we have fitted a single exponential decay model to the measured fluorescence decays as a method for determining the mean fluorescence decay time and to provide useful contrast in the fluorescence lifetime images. The measurement of fluorescence lifetimes in biological tissue is always complicated by the presence of multiple fluorophores that can each be present in a range of different microenvironments. As a result, the fluorescence decay profiles from each pixel in the image are complex and not accurately described by a single exponential decay model. Double or triple exponential models would provide better fits and have been used by some authors when analysing lifetime data of biological tissue autofluorescence. However, this 

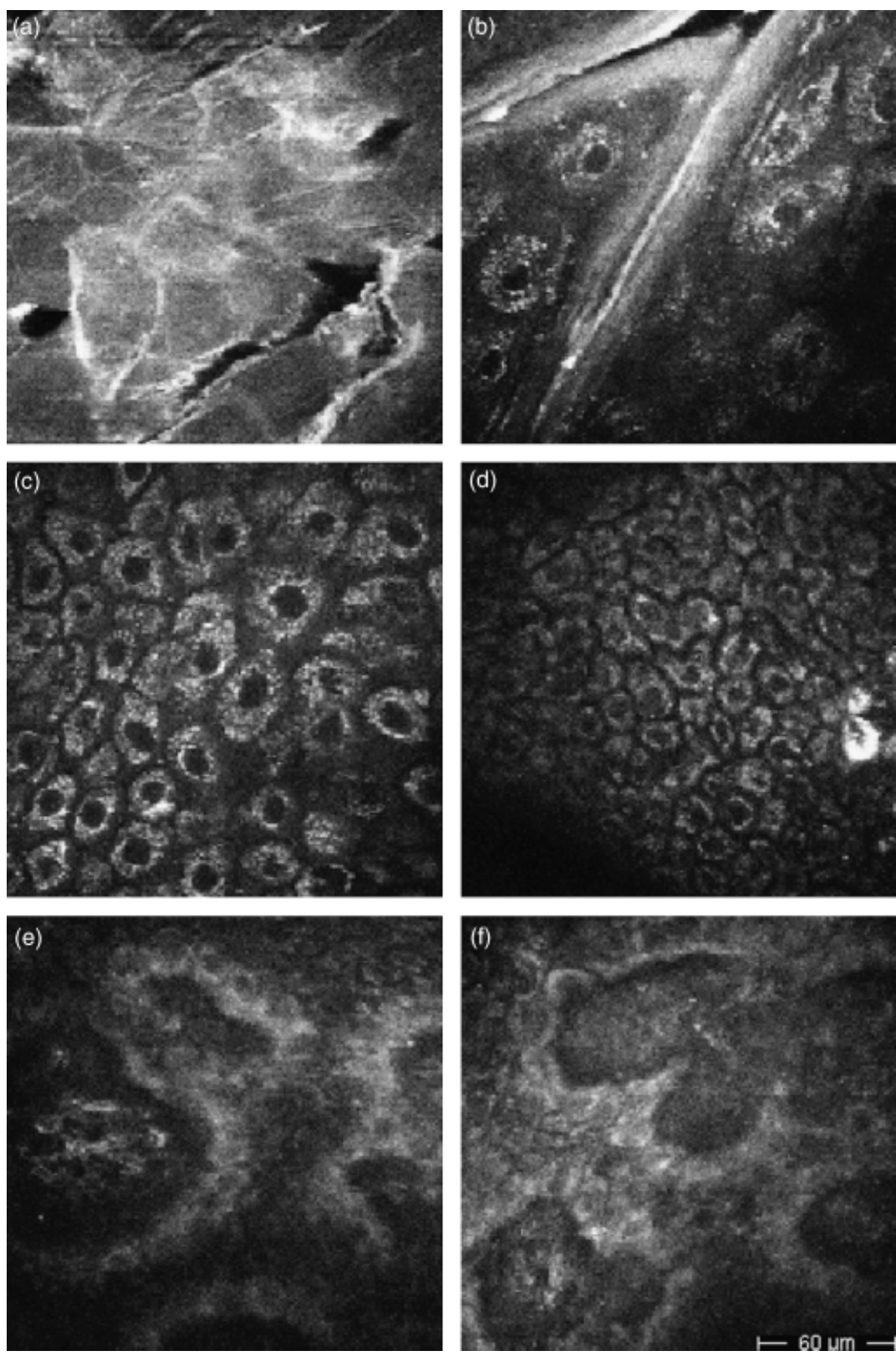

Fig. 4. ( $a, b, c, d, e, f)$ Intensity images of the epidermis; (a) stratum corneum (depth $0 \mu m$ ), (b) shift from the stratum corneum to the stratum

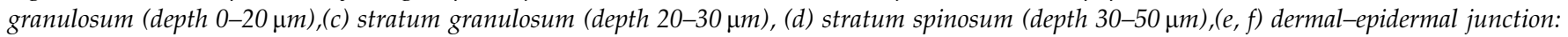
cells of the basal layer surrounding the papillae; (e)depth 50-80 $\mu \mathrm{m}$, (f) depth 80-90 $\mu \mathrm{m}$.

improved fit is often only the consequence of the higher number of fitting parameters used and it is no more realistic to assume the presence of only two or three mono-exponentially decaying fluorophores (or a single fluorophore with two or three discrete decay constants) in each pixel of the entire FLIM map than it is to assume the presence of a single fluorophore. Fitting multiexponential decays is more vulnerable to noise artefacts and requires a higher number of detected photons (i.e. longer data acquisition times) and a longer computation time compared with fitting a single exponential decay curve to the data. Therefore, the lifetime data presented in this paper represent a robust method to empirically determine the mean fluorescence decay time. More complex fluorescence decay models and analysis techniques will be investigated in future studies.

As humans begin to age, the skin inevitably becomes thinner, dry and rough, as a result of reduced metabolic activity and functional deficiency. Aged epidermis displays altered drug permeability, increased susceptibility to irritant contact dermatitis and often xerosis. The abnormality in permeability barrier homeostasis is 
accentuated further in photaged skin. The biochemical basis is a global reduction in skin lipids and profound abnormalities in cholesterol synthesis, particularly due to reduced activities of ceramide-generating enzymes $(20,21)$. In the elderly, lamellar bilayers display normal substructure and dimensions, but are focally lower in number, with reduced secretion of lamellar body contents $(20,21)$. Investigating the skin of elderly individuals by means of multiphoton tomography and FLIM, we demonstrated that epidermal cells show morphologic alterations in shape and size and appear more sparse. Moreover, fluorescence decay times are higher in the elderly, and this may correspond to a reduced metabolic activity (22), explaining the abovementioned impaired barrier function.

\section{Conclusion}

Since its introduction, MPM has contributed to great advances in different areas of biology and medicine. The non-invasive nature and enhanced penetration depth make MPM the preferred imaging modality in many fields of biomedical applications. In dermatology, applications such as the imaging and identification of skin tumours, especially BCC, seem to be extremely promising.

A major issue for an emerging imaging technique consists in the recognition of reference patterns, corresponding, in dermatological science, to the identification and characterization of healthy epidermis and dermis. The categorization of healthy cell types represents a pre-requisite for the distinction between healthy and pathological conditions, and in particular for the description of abnormal keratinocytes belonging to skin tumours of epidermal origin, such as basal cell and squamous cell carcinoma. Moreover, as epidermal morphology and function vary according to the skin site, an image database illustrating the diverse aspects of different skin sites has to be established, in order to orient the clinician coping with skin site variations of pathological conditions. This study provides quantitative and qualitative data on normal epidermis at different skin sites at different ages, and represents a reference for the clinician attempting to understand the effectiveness of multiphoton imaging in discriminating diseased states of the skin from normal ones.

\section{Acknowledgements}

The research leading to these results has received funding from the European Community's Seventh Framework Programme (FP7/2007-2013) under grant agreement no. 201577.

The authors wish to thank Doctor Rakesh Patalay from Imperial College London, UK, for assistance and helpful discussions relating to this work.

\section{References}

1. Tsai TH, Jee SH, Dong CY, Lin SJ. Multiphoton microscopy in dermatological imaging. J Dermatol Sci 2009; 56: $1-8$.

2. Leveque JL, Xhauflaire-Uhoda E, Pierard GE. Skin capacitance imaging, a new technique for investigating the skin surface. Eur J Dermatol 2006; 16: 500-506.

3. Masters BR, So PTC, Gratton E. Multiphoton excitation fluorescence microscopy and spectroscopy of in vivo human skin. Biophys J 1997; 72 : 2405-2412.

4. Zhuo S, Chen J, Jiang $X$, Cheng $X$, Xie S. Visualizing extracellular matrix and sensing fibroblasts metabolism in human dermis by nonlinear spectral imaging. Skin Res Technol 2007; 13: 406-411.

5. Lin SJ, Jee SH, Kuo CJ et al. Discrimination of basal cell carcinoma from normal dermal stroma by quantitative multiphoton imaging. Opt Lett 2006; 31: 2756-2758.

6. Paoli J, Smedh M, Ericson MB. Multiphoton laser scanning microscopya novel diagnostic method for superficial skin cancers. Semin $\mathrm{Cu}-$ tan Med Surg 2009; 28: 190-195.

7. Masters BR, So PTC, Gratton E. Optical biopsy of in vivo human skin: multi-photon excitation microscopy. Lasers Med Sci 1998; 13: 196203.

8. Layland K, Riemann I, Damour O, Stock UA, König K. Two-photon microscopes and in vivo multiphoton tomographs. Powerful diagnostic tools for tissue engineering and drug delivery. Adv Drug Deliv Rev 2006; 58: 578-596.

9. Malone JC, Hood AF, Conley T, Nurnberger J, Baldridge LA, Clendenon JL, Dunn KW, Phillips CL Three-dimensional imaging of hu- man skin and mucosa by twophoton laser scanning microscopy. J Cutan Pathol 2002; 29: 453-458.

10. König K, Riemann I. High-resolution multiphoton tomography of human skin with subcellular spatial resolution and picosecond time resolution. J Biomed Opt 2003; 8: 432439.

11. Dimitrow E, Riemann I, Ehlers A, Koehler MJ, Norgauer J, Elsner P, König K, Kaatz M. Spectral fluorescence lifetime detection and selective melanin imaging by multiphoton laser tomography for melanoma diagnosis. Exp Dermatol 2009; 18: 509-515.

12. Teuchner K, Freyer W, Leupold D, Volkmer A, Birch DJ, Altmeyer P, Stücker M, Hoffmann K. Femtosecond two-photon excited fluorescence of melanin. Photochem Photobiol 1999; 70: 146-151.

13. Vishwasrao HD, Heikal AA, Kasischke KA, Webb WW. Conforma- 
tional dependence of intracellular $\mathrm{NADH}$ on metabolic state revealed by associated fluorescence anisotropy. J Biol Chem 2005; 280: 25119-25126.

14. Tagami H. Functional characteristics of the stratum corneum in photoaged skin in comparison with those found in intrinsic aging. Arch Dermatol Res 2008; 300: S1-S6.

15. König K. Clinical multiphoton tomography. J Biophotonics 2008; 1: 13-23.

16. Paoli J, Smedh M, Wennberg AM, Ericson MB. Multiphoton laser scanning microscopy on non-melanoma skin cancer: morphologic features for future non-invasive diagnostics. J Invest Dermatol 2008; 128: 12481255.

17. Lin SJ, Jee SH, Dong CY. Multiphoton microscopy: a new paradigm in dermatological imaging. Eur J Dermatol 2007; 17: 361-366.

18. Dimitrow E, Ziemer M, Koehler MJ, Norgauer J, König K, Elsner P, Kaatz M. Sensitivity and specificity of multiphoton laser tomography for in vivo and ex vivo diagnosis of malignant melanoma. J Invest Dermatol 2009; 129: 1752-1758.

19. Skala MC, Riching KM, GendronFitzpatrick A, Eickhoff J, Eliceiri KW, White JG, Ramanujam N. In vivo multiphoton microscopy of NADH and FAD redox states, fluorescence lifetimes, and cellular morphology in precancerous epithelia. Proc Natl Acad Sci USA 2007; 104: 19494-19499.

20. Elias PM, Ghadially R. The aged epidermal permeability barrier: basis for functional abnormalities. Clin Geriatr Med 2002; 18: 103-120.
21. Reed JT, Elias PM, Ghadially R. Integrity and permeability barrier function of photoaged human epidermis. Arch Dermatol 1997; 133: 395-396.

22. Koehler MJ, Hahn S, Preller A, Elsner P, Ziemer M, Bauer A, König K, Bückle R, Fluhr JW, Kaatz M. Morphological skin ageing criteria by multiphoton laser scanning tomography: non-invasive in vivo scoring of the dermal fibre network. Exp Dermatol 2008; 17: 519-523.

\section{Address:}

Dr Elisa Benati

Department of Dermatology

University of Modena and Reggio Emilia

41124 Italy

e-mail:84498@studenti.unimore.it 